\title{
IDENTIFICAÇÃO DAS ESPÉCIES PARASITADAS POR Molothrus bonariensis NO CAMPUS II DA UNIVERSIDADE DO OESTE PAULISTA - UNOESTE
}

\author{
Marcelo Wendeborn Miranda de Oliveira ${ }^{1}$
}

\author{
Luiz Waldemar de Oliveira²
}

\author{
Victor Barro Franco ${ }^{3}$
}

\section{RESUMO}

O Molothrus bonariensis é uma ave passeriforme da família Icteridae, o macho adulto tem cerca de 20 $\mathrm{cm}$ e penas azuladas quase negras, a fêmea apresenta coloração marrom escura. Vários nomes populares são atribuídos a esta espécie, como vira-bosta, maria-preta, melro e chupim, usado até como um apelido pejorativo. Como hábito reprodutivo põe seus ovos em ninhos de outras espécies, isto por ter perdido sua capacidade de fabricar ninhos e cuidar da prole. Mais de cinquenta espécies podem ser parasitadas por esta ave. O objetivo deste trabalho foi identificar quais espécies são hospedeiras do Molothrus bonariensis nos recintos do Campus II da UNIVERSIDADE DO OESTE PAULISTA - (UNOESTE), assim como o período do ano em que ocorre este comportamento associado às espécies parasitadas. No período de observação foram registradas nove espécies sendo parasitadas, vistas oferecendo alimento ao ninhego de Molothrus bonariensis. Três das espécies pautadas, não foram encontradas em literatura.

PALAVRAS-CHAVE: Nidoparasita, Avifauna, Pássaro-preto.

\section{IDENTIFICATION OF SPECIES PARASITIZED BY Molothrus bonariensis II CAMPUS OF THE UNIVERSITY OF WESTERN SAO PAULO - UNOESTE}

\footnotetext{
ABSTRACT

The Molothrus bonariensis is a passerine bird in the Icteridae family, the adult male is about $20 \mathrm{~cm}$ and blue feathers almost black, the female has dark brown color, several popular names are assigned to this species, as he

1 Biólogo, Alta Sorocabana/APTA, Bolsista FAPESP.

${ }^{2}$ Biólogo, Me. Universidade do Oeste Paulista/UNOESTE.

${ }^{3}$ Biólogo, Bacharelado.

E-mail. marcelo_wendeborn@hotmail.com

E-mail. oliveira@unoeste.br

E-mail.rotciv182@msn.co
} 
had seen a shirt, black-maria, blackbird and Chupim, even used as a derogatory nickname. The reproductive habits of this bird, is by its eggs in the nests of other species, that having lost their ability to make nests and taking care of offspring. More than fifty species parasitized by this bird. The objective of this study is to identify which species are hosts in the precincts of the Campus II - UNOESTE Universidade do Oeste Paulista, and the period of the year in which this behavior occurs assciated species parasitized. In the observation period were recorded nine species being parasitized seen offering food to the nestling of Molothrus bonariensis. Three species were not found in guided literature.

KEY-WORDS: Nidoparasita, Avifauna Bird-black.

\title{
IDENTIFICACIÓN DE ESPECIES PARASITADOS POR Molothrus bonariensis CAMPUS DE LA UNIVERSIDAD DE SÃO PAULO II OESTE - UNOESTE
}

\begin{abstract}
RESUMEN
El Molothrus bonariensis es un ave paseriforme de la familia Icteridae, el macho adulto es de unos $20 \mathrm{~cm}$ y plumas negras casi azulados, la hembra tiene coloración marrón oscuro. Varios nombres populares son asignados a esta especie, como turd callejero, negro maria, mirlo y Chupim utilizan como un apodo peyorativo. Como hábito reproductiva pone sus huevos en los nidos de otras especies, es decir, han perdido su capacidad de fabricar nidos y cuidar a sus crías. Más de cincuenta especies pueden ser parasitados por este pájaro. El objetivo de este estudio fue identificar qué especies son Molothrus bonariensis anfitrión en el recinto del Campus II UNIVERSIDAD DE OCCIDENTE PAULO - (UNOESTE), y el período del año en el que se produce este comportamiento asociado con especies parasitadas. En este período de nueve especies que se están parasitados se registraron, con vistas a los alimentos a Molothrus bonariensis enclavado. Tres guiadas las especies que no se encuentran en la literatura.
\end{abstract}

PALABRAS-CLAVE: Nidoparasita, Avifauna, Pájaros-negro.

\section{INTRODUÇÃO}

O Brasil possui uma das mais ricas avifaunas do mundo com 1822 [+31] espécies segundo CBRO, 2011. A Amazônia apresenta o maior número de espécies, seguida pela Mata Atlântica e o Cerrado; entretanto, a maioria das espécies endêmicas do Brasil é encontrada na Mata Atlântica, especialmente nas terras baixas do litoral Sudeste e no Nordeste, 92\% das aves brasileiras são espécies residentes, sendo apenas 8\% espécies migrantes (SICK, 1993).

O Molothrus bonariensis (figura 1) pertence à Ordem Passeriforme e a Família Icteridae, conhecido popularmente como chupim, chopim, vira-bosta, maria-preta, azulego e outras denominações regionais. Pesando entre 42 e $52 \mathrm{~g}$ e medindo entre 
Volume 10, Número 3, 2014

Biodiversidade e Unidades de Conservação

16,5 e $21,5 \mathrm{~cm}$ de comprimento, o macho adulto é preto-azulado e a fêmea adulta é marrom-escura. É um parasita de ninhos de outras espécies, tais seres dependem inteiramente do hospedeiro para se reproduzir, pois perdeu tanto a habilidade de fazer o próprio ninho como o instinto de cuidar da prole. (SOUZA, 1998; SICK, 1997).

Figura 1 - Molothrus bonariensis macho. - Campus II - UNOESTE, Presidente Prudente.

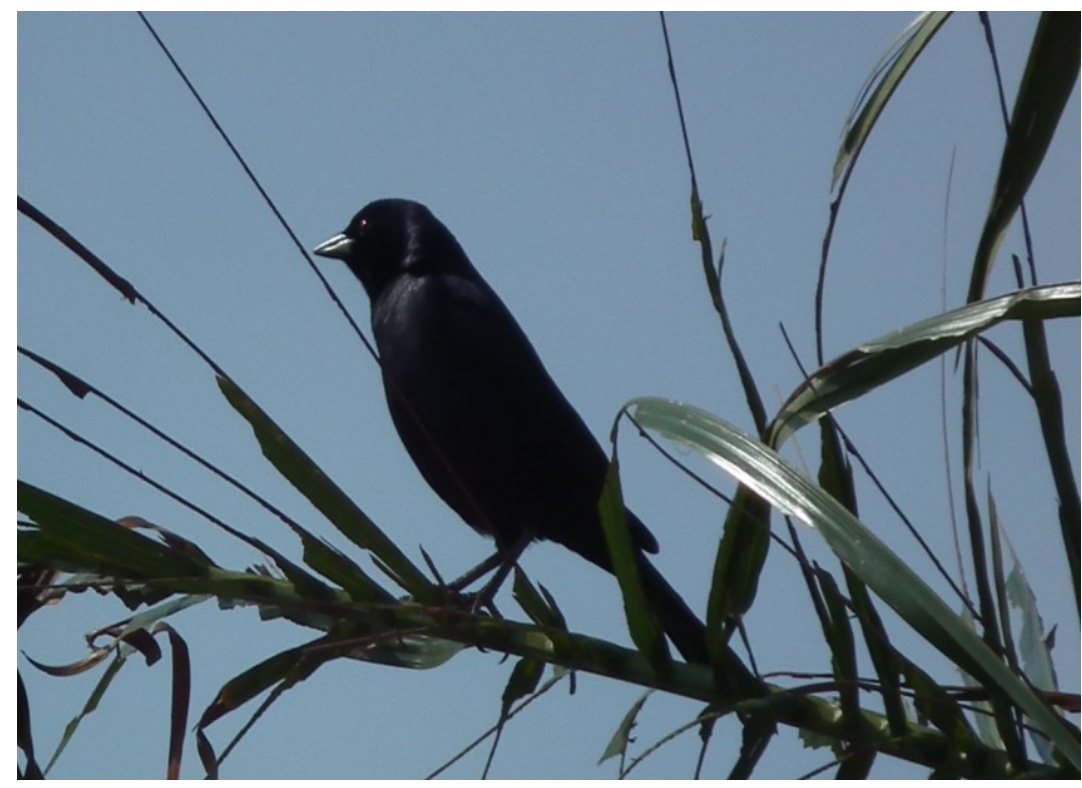

Fonte: Johnny Michael, (2012).

O parasitismo de ninho é uma estratégia reprodutiva onde o parasita, põem seus ovos em ninhos de indivíduos de outras espécies, os hospedeiros, que fornecem todo o cuidado parental aos ninhegos (SICK, 1997).

Cerca de $1 \%$ do total de espécies de aves são parasitas de ninho. Estes incluem representantes de Indicatoridae. Quase a metade das 130 espécies de Cucullidae, dois gêneros de Ploceidae (Vidua e Anomalospiza), cinco espécies de Icteridae e uma de Anatidae (Heteronetta atricapilla), entre outros. A maioria das aves hospedeiras são nutrizes (altriciais), isto é, os ninhegos e jovens parasitas dependem delas para alimentar-se (FORNERIS, 1998).

O impacto do parasitismo sobre o hospedeiro pode ser calculado pela proporção de ninhos parasitados localmente e pelo comportamento do parasita em eliminar os ovos do hospedeiro ou deste abandonar o ninho deixando de chocar ou 
eliminar os ovos do parasita. Molothrus bonariensis diminui a capacidade reprodutiva dos hospedeiros, pois foi observado que estes podem quebrar ou jogar fora do ninho os ovos da espécie parasitada (FORNERIS, 1998; REBOREDA et al., 2003).

O Chopim habita paisagens semiabertas, campos de cultura, pastos e áreas urbanas e ocorre do Panamá e Antilhas, através da maior parte da América do Sul, até a Argentina e o Chile e em todas as regiões do Brasil (SICK, 1997). Molothrus bonariensis expande seu território, tendo alcançado a Flórida em 1991 quando sua presença foi confirmada ao norte de Maine (POST e CRUZ, 1993), no Yucatã, México, no arquipélago das Bahamas (BALTZ, 1995), em maio de 1996 (KLUSA, 1998), trazendo sérios riscos às espécies endêmicas da região.

Molothrus bonariensis é um parasita extremamente generalista na seleção de seus hospedeiros, seu ovo já foi encontrado em ninhos de 243 espécies. A fêmea se aproveita de algum momento de distração do possível hospedeiro e deposita seus ovos no ninho, portanto, menos da metade das posturas coincide com o início da postura de seus hospedeiros, esse é um dos motivos pela qual a maioria de seus ovos não eclode, além deste, os ninhos podem ser abandonados, ou ainda os ovos do parasita são destruídos pelo hospedeiro (KATTAN, 2002).

Por causa de seu comportamento peculiar, Molothrus bonariensis tornou-se uma ave de relevância, sendo alvo de muitos estudos, o seu padrão comportamental foi estudado por Porto e Piratelli, (2005) e sua biometria, muda e placa de incubação por Bugoni et al. (2002). Já Pereira et al. (2001) coletaram sangue e isolaram o vírus ilhéus, transmissível a outros animais, inclusive ao ser humano. A presença de Plasmodium sp foi relatada por Paraense, (2004).

Assim como dezenas de outras aves brasileiras, Molothrus bonariensis foi alvo de tráfico no Rio Grande do Sul (FERREIRA; GLOCK, 2004; ARAÚJO et al., 2010) e em Pernambuco (PEREIRA; BRITO, 2005).

Raras são as localidades no território Brasileiro onde Molothrus bonariensis não é encontrado, pois a espécie se disseminou muito bem no país. Há vários trabalhos direcionados ou citando o $M$. bonariensis em inúmeras regiões distintas como, na Estação Ecológica de Carijós (ESEC), Florianópolis, Santa Catarina (EFE et 
al., 2007), e no Parque Estadual da Fonte Grande, Vitória, Espírito Santo (SIMON et al., 2007). Na maioria dos levantamentos de avifauna, os autores relataram a presença de Molothrus bonariensis pelo território brasileiro, nos municípios de Brasília, Distrito Federal (CAVALCANTI; PIMENTEL, 1988; BORGES, 2008), Goiânia, Goiás (MONTEIRO; BRANDÃO, 1995), São Paulo, São Paulo (FORNERIS, 1998; PEREIRA et al., 2001), Florianópolis, Santa Catarina (SOARES, 2004), Seropédica, Rio de Janeiro (GABRIELE e PIRATELLI, 2005), Rio Grande, Rio Grande do Sul (VOTTO et al., 2006), Sorocaba, São Paulo (SILVA; NAKANO, 2008), Frederico Westphalen, Rio Grande do Sul, (TEIXEIRA et al., 2009), e no município de Macapá, Amapá, (AGUIAR; NAIFF, 2010).

Onde existem indivíduos da espécie Molothrus bonariensis, é possível observar o parasitismo de ninho, pois esta ave não é um parasita específico como Molothrus rufoaxillari (D'ANGELO NETO et al., 2006).

No Brasil, em 1988, Cavalcanti e Pimentel, relataram à dispersão do Molothrus bonairensis, sendo encontrados como hospedeiros desta espécie o Mimus saturninus, Thraupis sayaca, Thraupis palmarum, Cypsnagra hirundiacea, Hemithraupis guira, Ammodramus humeralis, Tyrannus savana e Neothraupis fasciata, esta, nunca parasitada em seu habitat natural.

Forneris (1998) relata um aumento na população de Molothrus bonariensis que freqüenta a Cidade Universitária "Armando de Salles Oliveira”, Butantã, São Paulo, ele identificou apenas Zonotrichia capensis, como hospedeira neste local. Figueiredo (1995), em sua revisão relata parasitismo em Xalmis irupero, Machetormis rixosus, Troglodytes aedon, Passer domesticus, Sicalis flaveola;

Foi observado por Borges (2008), que o parasitismo ocorreu apenas em áreas alteradas de cerrado, em ninhos de Turdus amaurochalinus, Thraupis sayaca, Coryphospingus pileatus, Neothrupis fasciata, Piranga flava e Zonotrichia capensis.

Em Porto Rico, Pérez-Rivera (1986) estudou o parasitismo de ninhos por Molothrus bonariensis sobre as espécies Zenaida aurita, Columbiana passerina, Mimus polyglotus, Tyranus dominicensis, Vireo latimeri, Vireo altiloquus, Icterus dominicensis, Quiscalus Níger, Tiaris bicolor e Lonchura punctulata. Na Argentina, 
Reboreda et al. (2003) estudaram o parasitismo sobre Pseudoleistes virescens. $\mathrm{Na}$ Colômbia, Mezquida (2003) observou na espécie Diuca diuca e Anhumada (2001) observou o parasitismo sobre as espécies Thryophilus rufalbus e Thryophilus leucoti., No Chile Marín (2000) observou o parasitismo em 15 espécies, sendo apenas 6 delas capazes de criar os ninhegos.

O objetivo deste trabalho foi identificar quais espécies são hospedeiros de Molothrus bonariensis, nos recintos do Campus II da Unoeste - Universidade do Oeste Paulista, assim como o período do ano em que ocorre este comportamento associado às espécies parasitadas.

\section{DESENVOLVIMENTO}

\subsection{Material e Método}

O estudo foi realizado no Campus II da UNOESTE (Universidade Oeste Paulista), situado no município de Presidente Prudente /SP, Rodovia Raposo Tavares, Km 572, bairro Limoeiro com coordenadas geográficas ( $22^{\circ} 07^{\prime} 02.33^{\prime \prime} \mathrm{S}$ e $51^{\circ} 27^{\prime} 03.72$ " O) (Figura 2).

O método utilizado para a realização deste trabalho foi o da observação direta, a olho desarmado ou com o uso de binóculos, onde após confirmarmos a presença do filhote de chupim, através de sua vocalização, acompanhávamos este filhote voando para encontrar seus pais (hospedeiros), ou esperávamos os hospedeiros chegar até o filhote, para alimentá-lo. Após a observação, registrava qual era a espécie parasitada. 
FIGURA 2: Imagem de satélite do Campus II da UNOESTE de Presidente Prudente/SP

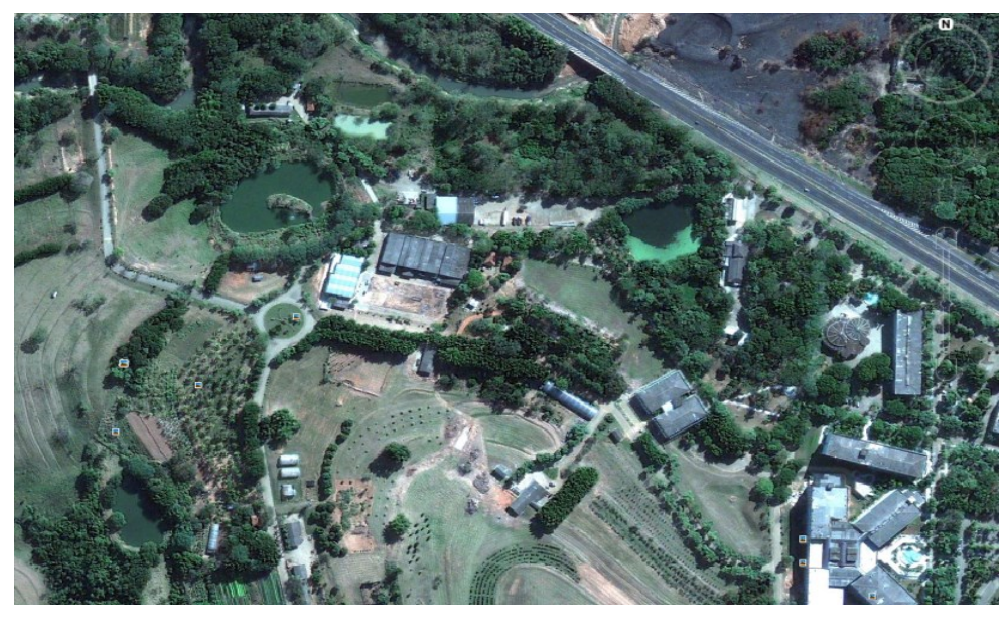

Fonte: Google Earth, (2012).

Após uma análise dos diversos ambientes presentes no campus, estabeleceuse uma trilha que abrangesse a maior quantidade dentre estes diversos ambientes, onde a sequência de áreas percorridas (figura 3 ) foram as seguintes 03, 04, 05, 06, $07,16,18,25,20,21,22,23,24$, e retornando pelas áreas $23,22,21,20,18,17,05$, 04 e 03. Neste trajeto, estabelecemos pontos de observação, o primeiro próximo às áreas 04,05 e 06 (ponto 1 - próximo à torre de cristal), o segundo próximo à área 18 (ponto 2 - viveiro de mudas), o terceiro na área 25 (ponto 3 - área da piscicultura e córrego limoeiro), o quarto próximo às áreas 20,21, 22, 23 e 24 (ponto 4 - chácara da zootecnia) e o quinto ponto próximo às áreas 16 e 17 (ponto 5 - serpentário)

O ponto 1 é constituído por edificações, estacionamento calçado, gramados e várias espécies arbóreas e herbáceas. O ponto 2 é formado por pomar de goiabeiras, acerolas, pequenos bosques de espécies nativas e exóticas, variedade grande de herbáceas, gramados, estacionamento calçado e edificações. O ponto 3 é constituído por um ribeirão possuindo mata ciliar secundária, área da piscicultura com tanques cheios e vazios, bosques, gramados e um pequeno brejo. O ponto 4 é formado por estábulo, suinocultura, edificações, estacionamento calçado, bosque e pastagem. $\mathrm{O}$ ponto 5 é constituído por uma lagoa artificial, bosques de espécies nativas e exóticas e estacionamento calçado. 
Figura 3: Esquema representando os diferentes setores do Campus II da UNOESTE de Presidente Prudente/SP.

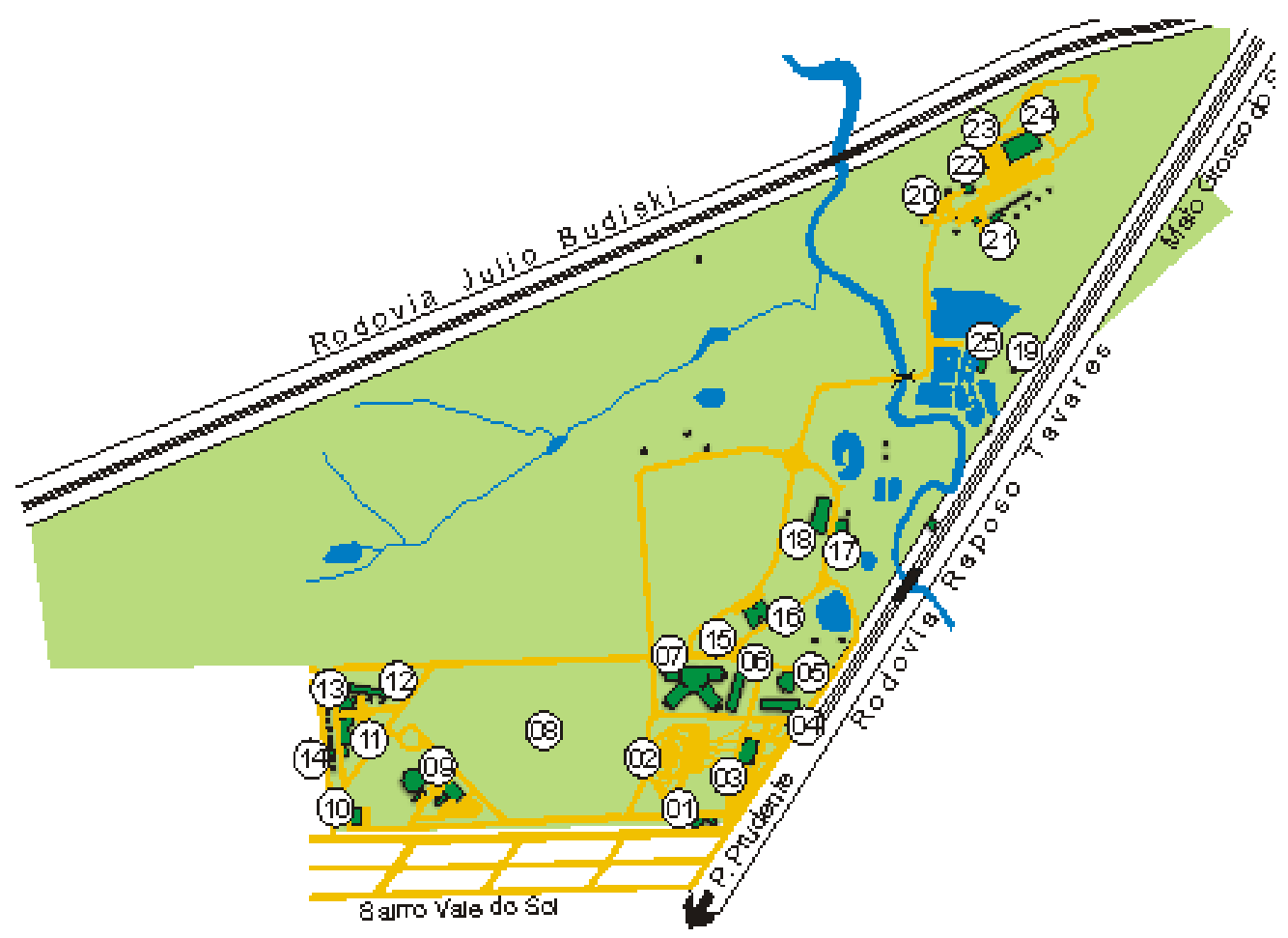

\begin{tabular}{|c|c|c|}
\hline \multicolumn{3}{|c|}{ LEGENDA } \\
\hline 01 Prédio dos Funcionários & 08 Centro Olimpico & 17 Posto e Central de véiculos II \\
\hline 02 Igreja & 09 Hospital vetem inário & 18 Serralheria e Wurcenaria \\
\hline 03 Salấo de Festas & 10 Canil & 19 Residência p ara Funcionários \\
\hline 04 Bloco de Engenharia, & 11 Amoxarifado & 20 Leiteira \\
\hline Administraçẫo e Contábeis & 12 Fábrica de Lajotas & 21 Pocilga \\
\hline 05 Restaurante Torre de Cristal & 13 Pátio de Carpintaria e Ammação & 22 Residência para Funcionários \\
\hline 06 Prédio de Pós-graduação & || 14 Residência para Funcionários & 23 Quadra de Bocha \\
\hline 07 Bloco Corrunicação. & 15 Praça Oriental & 24 Campo de Futebol Súço \\
\hline Psicologia e Direito & 16 Bloco de Agronomiaßeterinária & 25 Piscicultura \\
\hline
\end{tabular}

Fonte: http://www.unoeste.br/site/auniversidade/imagens/Campus-g-2.gif, (2012).

O percurso foi realizado à pé, em velocidade constante e com paradas de 20 minutos em cada ponto. As visitas ocorreram uma a duas vezes por semana, entre 4 e 5 horas por dia de esforço, sempre iniciando entre as 7:00 e 8:00 horas, e se estendendo entre 11:00 e 13:00 horas. As observações ocorreram durante os meses 
de outubro, novembro e dezembro de 2011, janeiro, fevereiro, março, abril e maio de 2012, totalizando 144 horas de observações.

Por se tratar de um trabalho preliminar que terá prosseguimento, os dados obtidos não sofrerão nenhum tipo de tratamento estatístico.

\subsection{Resultados e Discussão}

Tabela 1 - Espécies parasitadas por Molothrus bonariensis, observadas no Campus II da Unoeste, em Presidente Prudente, durante o período reprodutivo.

\begin{tabular}{lccc}
\hline \multicolumn{1}{c}{ FAMÍLIA } & NOME CIENTíFICO & NOME VULGAR & TAMANHO \\
\hline Tiranidae & Pitangus sulphuratus & bem-te-ví & $22,5 \mathrm{~cm}$ \\
Tiranidae & Tirannus melancolicus & Suiriri & $21,5 \mathrm{~cm}$ \\
Mimidae & Mimus saturninus & sabiá do campo & $26 \mathrm{~cm}$ \\
Turdidae & Turdus leucomelas & sabiá de barranco & $22 \mathrm{~cm}$ \\
Turdidae & Turdus amaurochalinus & sabiá poca & $22 \mathrm{~cm}$ \\
Thraupidae & Thraupis sayaca & sanhaçu cinzento & $17,5 \mathrm{~cm}$ \\
Thraupidae & Conirostrum speciosum & figuinha do rabo castanho & $10,5 \mathrm{~cm}$ \\
Vireonidae & Cyclarhis gujanensis & Pitiguarí & $16 \mathrm{~cm}$ \\
Emberizidae & Zonotrichia capensis & tico tico & $15 \mathrm{~cm}$ \\
\hline
\end{tabular}

Fonte: Os Autores, (2012).

No campus II da UNOESTE o Molothrus bonariensis é uma ave classificada como residente, estando presente em todos os meses em que ocorreram as observações, e em mais de $90 \%$ do total das visitas realizadas, este dado coincide com a maior parte dos trabalhos realizados em outras regiões do país e de outros países quando envolve o levantamento da avifauna dos locais estudados e a sazonalidade na frequência das espécies (CAVALCANTI; PIMENTEL, 1988; MONTEIRO; BRANDÃO, 1995; FORNERIS, 1998; MARÍN, 2000; PEREIRA et al., 2001; SOARES, 2004; MALLET-RODRIGUES, 2005; PORTO; PIRATELLI, 2005; VOTTO et al., 2006; BORGES, 2008; SILVA e NAKANO, 2008; TEIXEIRA et al., 2009; AGUIAR; NAIFF, 2010). 
Foi observado que indivíduos de Molothrus bonariensis, são mais freqüentes e em maior quantidade, em áreas com maior interferência humana, (áreas 03, 04, 05, 06, 07, 20, 21, 22, 23 e 24 da figura 3), se alimentando de restos de alimentos e/ou alimentos ofertados aos animais domésticos, ou ainda revirando excrementos de outros animais, comportamento também observado por Porto e Piratelli (2005). Portanto, sendo uma espécie extremamente adaptada a ambientes alterados (CAVALCANTI; PIMENTEL, 1988; FRANÇA; MARINI, 2009), assim como é a maior parte da área existente no CAMPUS II da UNOESTE. Onde existem poucas áreas com a presença maciça de espécies arbóreas, formando um "bosque", ou uma mata ciliar, e tampouco a presença de mata nativa, o que dificultaria o parasitismo (LEGAL, 2007).

Dentre as 9 espécies parasitadas por Molothrus bonariensis observadas no Campus II, 6 são citadas foram Sick (1997), mas as outras 3 espécies (Cyclarhis gujanensis, Conirostrum speciosum e Turdus leucomelas), não encontrei relatos na literatura, em minhas pesquisas.

Este acréscimo de espécies pode estar associado ao fato de, o ambiente em estudo ser totalmente alterado, com presença de gramados, pastagens, pomares, áreas agrícolas, várias espécies vegetais exóticas, de caráter ornamental, pequenos bosques formados por espécies arbóreas nativas e exóticas, expondo assim, mais espécies a ação parasitária do Chopim, comprometendo seriamente a reprodução e reposição de indivíduos das espécies parasitadas de acordo com estudos realizados em outras regiões. Fato observado e confirmado por outros autores (CAVALCANTI; PIMENTEL, 1988; FORNERIS, 1998; FRANÇA; MARINI, 2009; KLUSA 1998; REBOREDA et al., 2003).

A presença de filhotes parasitas foi observada até o mês de fevereiro de 2012 , quando observamos um único filhote de Molothrus bonariensis sendo alimentado por uma fêmea de Pitiguarí. Esta observação coincide com outros autores, pois este é o período reprodutivo das aves encontradas em regiões tropicais (FORNERIS, 1998; REBOREDA et al., 2003; FIORINI et al., 2005; LEGAL, 2007; RUBIO; PINHO, 2008). 
Em todas as vezes que presenciamos o parasitismo, o hospedeiro sempre estava alimentando apenas um filhote de Molothrus bonariensis e nenhum filhote próprio, fato este que pode estar relacionado ao que afirma Forneris (1998) e Reboreda et al. (2003), onde é possível que ao visitar os ninhos, Molothrus bonariensis pode quebrar o ovo do hospedeiro ou até mesmo, o jogar para fora do ninho.

Foi observado uma variação em tamanho das espécies parasitadas, onde os menores indivíduos afetados são das espécies Conirostrum speciosum, Cyclarhis gujanensis, Zonotrichia capensis e Thraupis sayaca, e os maiores Pitangus sulphuratus, Tirannus melancolicus, Mimus saturninus, Turdus leucomelas e Turdus amaurochalinus (Tabela 1). Estas observações coincidem com os estudos de Fiorini et al. (2005) que relatam o sucesso reprodutivo do Molothrus bonariensis, em hospedeiros de diferentes tamanhos corporais, quando observou o parasitismo em Troglodytes musculus, espécie onde os indivíduos alcançam $12 \mathrm{~cm}$ de comprimento.

De acordo com Rodrigues (2005), Turdus leucomelas (sabiá barranco), Turdidae, pode ter seu ninho predado por Corruíra, Troglodytes musculus (Troglodytidae), que se alimenta dos ovos. A literatura consultada não apresentou dados sobre o parasitismo de ninho da espécie por Molothrus bonariensis.

Com relação à espécie Mimus saturninus (sabiá do campo), Mimidae, as observações foram semelhantes às relatadas na literatura. $E$ outubro, período reprodutivo, encontramos adultos tratando de um filhote de Molothrus bonariensis, e em dezembro, um casal alimentando uma ninhada de 3 filhotes da própria espécie. Estas observações estão de acordo com Rodrigues (2009), que afirma que a espécie se reproduz de setembro a dezembro, apresenta a postura variando de um a seis ovos, mas nunca com mais de três filhotes. O Mimus saturninus é vítima de parasitismo por Molothrus bonairensis na Argentina (REBOREDA et al., 2003; FIORINI et al., 2005), em Brasília (CAVALCANTE; PIMENTEL, 1988; RODRIGUES, 2009).

Ocorrências semelhantes identificam em Turdus amaurochalinus (sabiá poca), Turdidae, equivalente às relatadas por Reboreda et al. (2003), Borges (2008) e Fiorini 
et al. (2005) que ainda observou que o parasitismo foi mais acentuado em Mimus saturninus.

Thraupis sayaca (sanhaçu-cinzento), Traupidae, espécie comum em áreas abertas arborizadas, equivalente às áreas observadas no Campus II, também foi parasitada por Molothrus bonairensis no Distrito Federal (CAVALCANTE; PIMENTEL, 1988; BORGES, 2008) sendo o parasitismo mais acentuado em áreas alteradas.

A espécie Zonotrichia capensis é uma das espécies preferidas como hospedeira pelo Molothrus bonariensis, inclusive em outros países (FORNERIS, 1998; MEZQUIDA, 2003; REBOREDA et al., 2003; BORGES, 2008).

Algumas espécies componentes da avifauna do Campus II da UNOESTE, não foram parasitadas na temporada reprodutiva em estudo, são apontadas como hospedeiras de ninhegos de Molothrus bonairensis: Passer domesticus Sicalis flaveola (FIGUEIREDO, 1995; MARIN, 2000), columbiformes (Columbina talpacoti, Scardafella squamata e patagioenas picazuro) (BORGES, 2008), Vale salientar que todas estas espécies apontadas se reproduziram normalmente na área em estudo, sendo que os columbiformes apresentaram reprodução em todos os meses de observação, sempre construindo ninhos e cuidando de seus próprios filhotes.

Se o parasitismo acentuado pelo Molothrus bonairensis chegar a prejudicar a reprodução de alguma das espécies de aves presentes no campus II da Unoeste, a ponto de levar a um declínio digno de extinção local, um manejo deverá ser estudado para a preservação local das espécies parasitadas, como retirada dos ovos do parasita dos ninhos hospedados.

\section{CONCLUSÃO}

$\mathrm{Na}$ área estudada a ação do Molothrus bonariensis foi extremamente prejudicial à espécie hospedeira, pois em nenhuma das observações o hospedeiro estava alimentando algum de seus filhotes além do ninhego parasita.

A maioria das espécies parasitadas são as mesmas observadas em estudos semelhantes. 
$\mathrm{Na}$ estação reprodutiva em estudo, não ocorreu parasitismo de algumas espécies que costumam ser parasitadas em outras áreas, como o Passer domesticus, Sporophila caerulescens, Sicalis flaveola e os Columbifomes. A presença de espécies hospedeiras, que não encontrei listadas na literatura pode ser atribuída ao fato do ambiente em estudo, ser totalmente antrópico, praticamente reconstruído, o que levou a uma maior exposição destas espécies a ação do parasita.

\section{REFERÊNCIAS}

AGUIAR K.M.O.; NAIFF, R.H. Composição da avifauna da Área de Proteção Ambiental do Rio Curiaú, Macapá, Amapá, Brasil. Ornithologia. v.4, n.1, p.36-48. 2010.

ANHUMADA, J. A. Comparison of the reproductive biology of two Neotropical wrens in an unpredictable environment in Northeastern Colombia. The Auk., v.118, n.1, p.191-210. 2001.

ARAUJO, A.C.B.E. et al. Diagnóstico sobre a avifauna apreendida e entregue espontaneamente na Região Central do Rio Grande do Sul, Brasil. R. bras. Bioci. Porto Alegre,. v.8, n.3, p.279-284. 2010.

BALTZ, M. First Records of the Shiny Cowbird (Molothrus bonariensis) in the Bahama Archipelago. The Auk, v.112, n.4, p.1039-1041. 1995.

BORGES, F. J. A. Efeitos da fragmentação sobre o sucesso reprodutivo de Aves em uma área de cerrado no Distrito federal. 2008. Dissertação (Mestrado) - Universidade de Brasília.

BUGONI, L. et al. Biometry, molt and brood patch parameters of birds southern Brazil. Ararajuba,. v.10, n.1, p.85-94. 2002.

CAVALCANTI, R. B.; PIMENTEL, T. M. Shiny cowbird parasitism in central Brazil. The Condor, p.4043. 1988.

CBRO - Comitê Brasileiro de Registros Ornitológicos. 2008. Lista das Aves Brasileiras. Versão 05/10/2008. Disponível em: <(http://www.cbro.org.br/CBRO/lista br.htm)>. Acesso em: 13 abr. 2012.

CBRO - Comitê Brasileiro de Registro Ornitológico. 2010. Lista das Aves Brasileiras. Versão 25/01/2011. Acesso em: 28 jun. 2012

D'ANGELO NETO, S.; LUZ, G. R. D.; VIANNA, M. D. O. P. Observacoes sobre o parasitismo de Molothrus rufoaxillaris sobre Gnorimopsar chopi e outros aspectos de sua historia natural no norte de Minas Gerais: por que Agelaioides fringillarius nao e parasitado?. Unimontes Cientifica, v.8, n.1, p. 53-58. 2006.

EFE, M.A.; AZEVEDO, M.A.; FILIPPINI, A. Avifauna da Estação Ecológica de Carijós, Florianópolis SC. Ornithologia, v.2, n.1, p.1-13. 2007.

FERREIRA, C. M.; GLOCK, L. Diagnóstico preliminar sobre a avifauna traficada no Rio Grande do Sul, Brasil. Biociências,. v.12, n.1, p.21-30. 2004. 
FIGUEIREDO, L. F. A. A reprodução do joão-de-barro, Furnarius rufus (Gmelin, 1788): uma revisão. Boletim do CEO,. n.11, p.2-33. 1995.

FIORINI, REBOREDA et al. Éxito reprodutivo del tordo renegrido ( $M$. bonariensis) em hospedadores de distintos tamanõ corporal. Hornero, Argentina v.20, n.2, p.173-182. 2005.

FONERIS, L. Gaudério, agente de extinçao e ameaça à avifauna? Boletim do CEO, São Paulo, v.13, p.24-27. 1998.

FRANÇA, L. C.; MARINI, M. A. Teste do efeito de borba na predação de ninhos naturais e artificiais no Cerrado. Zoologia v.26, n.2, p.241-250. 2009.

KATTAN, G. H. Shiny cowbirds follow the "shotgun" strategy of brood parasitism. Animal Behaviour,. v.53, p.647-654. 1997.

KLUSA, D. First Record of Shiny Cowbird (Molothrus bonariensis) in Yucatãn, Mexico Wilson Bull, v.110, n.3, p.429-430. 1998.

LEGAL, E. Aspectos da nidificação do siriri, Tyrannus melancholicus em Santa Catarina, (Vieillot, 1819 Atualidades Ornitológicas On-line n.140, nov./dez., 2007 - Disponível em: <www.ao.com.br>. Acesso em: 27 abr. 2012.

MALLET-RODRIGUES, F. Molt-Breeding cycle in passerines from a foothill forest in southeastern Brazil. Revista Brasileira de Ornitologia,. v.13, n.2, p.155-160. 2005.

MARÍN, M. The shiny cowbird (Molothrus bonariensis) in Chile: introduction or dispersion? its hosts and parasitic trends. Ornitologia neotropical, v.11, 2000.

MARINI, M. Â. et al. Biologia reprodutiva de Tyrannus savana (Aves, Tyrannidae) em cerrado do Brasil Central. Biota Neotrop. v.9, n.1, 2009.

MEZQUIDA, E. T. La reproducción de cinco espécies de Emberizidae y Fringilidae em la Reserva de Ñacuñán, Argentina. Hornero, v.18, n.1, p.13-20, 2003.

MONTEIRO, M.P.; BRANDÃO, D. Estrutura da comunidade de aves do "campus Samambaia" da Universidade Federal de Goiás, Goiania, Brasil. Ararajuba, v.3, p.21-26, 1995.

PARAENSE, L. W. A Fragment of Malaria History. Mem Inst Oswaldo Cruz, Rio de Janeiro, v.99, n.4, p.439-442. June, 2004.

PEREIRA, G. A.; DE BRITO, M. T. Diversidade de aves silvestres brasileiras comercializadas nas feiras livres da Região Metropolitana do Recife, Pernambuco. Atualidades Ornitológicas, v.126, n.14, 2005.

PEREIRA, L. E. et al. Arbovírus Ilheus em aves silvestres (Sporophila caerulescens e Molothrus bonariensis) llheus arbovirus in wild birds (Sporophila caerulescens and Molothrus bonariensis). Rev. Saúde Pública, São Paulo. v.35, n.2, 2001.

PEREZ-RIVERA, R. A. Parasitism by the shiny cowbird in the interior parts of Puerto Rico. J. Field Ornitol. v.57, n.2, p.99-104. 1986.

PORTO, G. L.; PIRATELLI, A. Etograma da maria-preta, Molothrus bonariensis (Gmelin) (Aves, Emberizidae, Ecterinae). Revista brasileira de zoologia, v.22, n.2, p.306-312. 2005. 
POST, W.; CRUZ, A.; MCNAIR, D. B. The North American invasion pattern of the shiny cowbird. Journal of Field Ornithology, v.64, n.1, p.32-41, 1993.

REBOREDA, J. C. et al. Impacto del parasitismo de cría del tordo renegrido (Molothrus bonariensis) sobre el éxito reprodutivo de sus hospedadores. Hornero, Argentina, v.18, n.2, p.77-88. 2003.

RODRIGUES, F. M. Molt-Breeding cycle in passerines from a foothill forest in southeastern Brazil. Revista Brasileira de Ornitologia v.13, n.2, p.155-160 dez., 2005.

RODRIGUES, S.S. Biologia e sucesso reprodutivo de Mimus saturninus (aves - Mimidae) no cerrado. Dissertação (Mestrado). UNB - Brasília.

RUBIO, T.C.; PINHO, J.B. Biologia reprodutiva de Synallaxis albilora no Pantanal. Papéis Avulsos de Zoologia, v.48, n.17, 2008.

SICK, H. Birds in Brazil: a natural history. Princeton, EUA: Princeton University Press, 1993.

SICK, H. Ornitologia brasileira. Rio de Janeiro: Nova Fronteira, 1997. 862p.

SILVA, L.A.C.; NAKANO, C.A. Avifauna em uma Área de Cerrado no Bairro do Central Parque, Município de Sorocaba, São Paulo, Brasil. v.1, n.1, p.36-61. 2008.

SIMON, J.E.; LIMA, R.S.; CARDINALLI, T. Comunidade de aves no Parque Estadual da Fonte Grande, Vitória, Espirito Santo, Brasil. Revista Brasileira de Zoologia. v.24, n.1, p.121-132. 2007.

SOARES, A. G. A avifauna de uma Area no bairro Ribeirão da Ilha, Florianópolis, Santa Catarina: levantamento e implicações para a educação ambiental. Biotemas, v.17, n.2, p.107-124. 2004.

SOUZA D.G.S. Todas as aves do Brasil. Feira de Santana, Bahia: Dall, 1998. 258p.

TEIXEIRA, E.M.; BERNARDI, I.P.; JACOMASSA, F.A.F. Avifauna de Frederico Whestphalen, Rio Grande do SUL. Biotemas, v.22, n.4, p.117-124. 2009.

VOTTO, A.P. et al. Sazonalidade da avifauna no campus carreiros da fundação universidade federal do Rio Grande, Rio Grande do Sul, Brasil. Estud. Biol., v.28, n.62, p.45-55. 2006. 